\title{
Disjunction and Anaphora
}

\author{
Mandy Simons \\ Cornell University
}

\section{Introduction}

The goal of this paper is to account for the behavior of anaphora in the context of disjunction. There are two distinct sets of data to account for which, following Groenendijk and Stokhof (1990), I call "internal" and "external" anaphora. Internal anaphora is anaphora between disjuncts, the possibilities for which are illustrated in (1) and (2): ${ }^{1}$

(1) \#Either Jones owns a bicycle, or it's broken. ${ }^{2}$

(2) Either there's no bathroom in the house, or it's in a funny place.

External anaphora is anaphora between a disjunctive expression of any syntactic type, and a pronoun outside that expression, generally in a following sentence. Some examples are given in (3)-(5):

(3) Either a soprano or an alto will sing an aria. Then she will lead the audience in the national anthem.

(4) Jane either borrowed a car or rented a truck to get to Boston. \#It broke down on the way.

(5) Either a squirrel has got into the attic, or a bird is building a nest up there. We'll have to get it out.

Each set of data requires a somewhat different explanation, but both explanations will follow from quite natural assumptions about the function which disjunction fulfills in discourse.

\section{Internal Anaphora}

The internal anaphora data are well known from the literature, as is the puzzle they present. The puzzle, in brief, is this. Examples like (1) above indicate that indefinites in one disjunct cannot serve as antecedents to pronouns in another. In addition, it is generally assumed, on the basis of examples like (6), that indefinites in the scope of negation are inaccessible to following pronouns:

(6) George doesn't own a car. \#It's blue.

In "bathroom sentences" like (2), the indefinite is not only in a different disjunct to the pronoun, but also falls under the scope of negation. We would therefore 
expect anaphora between the two to be doubly bad; but in fact, it is perfectly acceptable.

Let me begin my explanation by making two additional observations. First, it is not the case that all anaphora is blocked by negation. Consider (7) and (8):

(7) This hospital has a maternity ward. It is very well run.

(8) This hospital does not have a maternity ward. It was closed due to lack of funds.

It is generally agreed that the anaphora in (8), where the indefinite falls under the scope of negation, is of a different type to the anaphora in (7). Nonetheless, it is clear that the pronoun in (8) is interpreted on the basis of the content of the first clause, in particular, of the indefinite NP.

The second observation is that the same kind of infelicity which occurs in (1) also occurs in the absence of anaphora. Compare (1), repeated here, with (9) and (10):

(1) \#Either Jones owns a bicycle, or it's broken.

(9) \#Either Jones owns a bicycle, or she owns a broken bicycle.

(10) \#Either Jones owns a bicycle, or she hides every bicycle she owns.

All of these seem equally peculiar, but we obviously cannot attribute the peculiarity of (9) and (10) to the impossibility of anaphora.

Note further that the infelicity of (1) seems different in kind to that of

(11) \#Every man ran away. He was afraid.

The pronoun in this example cannot be interpreted on the basis of the linguistic context. Naive speakers object to the sequence because the pronoun "doesn't refer to anything." In contrast, the pronoun in (1) does have an intuitively clear interpretation. Naive speakers agree that $i t$ is "the bicycle Jones owns." The problem with (1), as with (9) and (10), is that the disjunction as a whole just doesn't make any sense. Given the similarity between the anaphora and the nonanaphora cases, it is clearly desirable to give a single account for both.

This is what I will do here. In my account, I will suggest that anaphora of the type illustrated in (8) is freely allowed between disjuncts, but in cases like (1) results in disjunctions which are infelicitous for the same reason that (9) and (10) are: they violate a strong pragmatic constraint on disjunction.

\subsection{The Disjunction Constraint}

The function of a disjunctive sentence is to present alternatives. A disjunction offers characterizations of (at least) two different possible states of affairs, and asserts that at least one of these holds in the actual world. Now, reconsider example (9), repeated here: 
\#Either Jones owns a bicycle, or she owns a broken bicycle.

Note that the second disjunct of (9) entails the first. This means that whenever the world accords with the state of affairs described by the second disjunct, it also accords with the state of affairs described by the first. In other words, (9) does not present true alternatives; it does not present information about two distinct possible states of affairs. (9) seems to be ruled out because it does not do what disjunctions are supposed to do. To characterize this infelicitiy, let us say that disjunctions in which one disjunct entails the other are ruled out. ${ }^{3}$

This constraint is also responsible for the infelicity of the anaphora examples. Recall sentence (1), repeated here:

\section{\#Either Jones owns a bicycle, or it's broken.}

I noted above that intuitively, the pronoun in the second disjunct of (1) is interpreted as "the bicycle Jones owns." It is this intuition which the various "Etype" theories of pronoun interpretation attempt to capture. These theories, ranging from the syntactically constrained proposals made by Evans $(1977,1980)$ and developed in Neale (1990), to the "pragmatic" account suggested in Heim (1990) and developed in Stone (1992), all have a consequence in common. All of these theories assign to the pronoun in (1) an interpretation which entails the existence of a bicycle owned by Jones, and therefore that Jones owns a bicycle. Consequently, all of these theories predict that the second disjunct entails the first. Given the observations made above, this means that although the pronoun itself is interpretable, the disjunction as a whole is ruled out.

Let me make this explanation a little more concrete by introducing a formal framework. I adopt here the basic DRT framework of Kamp and Reyle (1993), into which I incorporate an E-type interpretation strategy. ${ }^{4}$ Following Kamp and Reyle, I represent disjunctions of the form [A or B] (where A and B may be expressions of any syntactic type) with a disjunctive condition of the form $\mathrm{K}_{1} \vee \mathrm{K}_{2}$, where each $\mathrm{K}_{\mathrm{n}}$ is itself a DRS. Accessibility relations and verification conditions are straightforward. I assume that discourse referents in the universe of any disjunct $\mathrm{K}_{\mathrm{n}}$ are inaccessible from any other, or from any superordinate condition, ${ }^{5}$ and that the verification conditions assign to or the truth conditions of inclusive disjunction.

Let us see, then, how the interpretation of (1) proceeds. At the first stage of DRS construction, a disjunctive condition is entered into the main DRS, and the content of each disjunct is entered into a sub-DRS. The first disjunct is reduced in the standard way, producing the DRS shown in (12):

$$
\text { [ II [x][bicycle }(\mathrm{x}) \text {, Jane owns } \mathrm{x}] \vee[\text { ][it's broken] ] }
$$

We now come to the pronoun in the second disjunct. What we expect to do is to use an old referent to translate it, so establishing an anaphoric link. But in this case, no such referent is accessible. We therefore employ a rescue strategy. We introduce a new referent, but we look for old information about some entity 
already in the discourse representation, and use that information to constrain the mappings from this new referent to individuals in the world. Formally, this is achieved by taking conditions on an existing discourse referent, and applying them to the new one. Thus, we satisfy the familiarity requirement of definites by using old conditions, instead of an old variable. Application of this strategy produces the complete DRS shown in (13):

\section{[ ][ $[\mathrm{x}][\operatorname{bicycle}(\mathrm{x})$, Jane owns $\mathrm{x}] \vee[\mathrm{y}][\operatorname{bicycle}(\mathrm{y})$, Jane owns $\mathrm{y}$, broken(y)]]}

My assumption here is that although discourse referents in one disjunct are inaccessible to pronouns in another, the informational content expressed by each disjunct is accessible, and can be used in constructing the representation.

The DRS in (13) shows clearly why the infelicitous disjunctions involving anaphora reduce to the non-anaphora cases in which one disjunct entails the other. (13) is, in fact, just the representation we would get for (9); the second disjunct of (13) entails the first. Thus, as I suggested above, the problem is not the interpretation of the pronoun, but the disjunction itself. In any disjunction of the form:

$$
\left[s \ldots P_{i}[-d e f] \ldots . .\right] \text { or }\left[s \ldots \text { pron }_{i} \ldots .\right]
$$

the interpretation strategy just outlined is the only one available. And in every such case, this strategy will give rise to the same kind of infelicity. The only exception to this is the case in which the indefinite falls under the scope of negation, that is, in bathroom sentences.

The interpretation of a bathroom sentence like (15) proceeds in just the same way. We introduce a disjunctive condition, and reduce the first disjunct. When we encounter the pronoun in the second disjunct, we look for an accessible discourse referent via which to interpret it. When we find that there is no accessible ref erent, we instead introduce a new referent, and constrain its ref erence by using old conditions: the conditions from the first disjunct. The negation, however, is not copied, as it is not part of a condition applied to a referent. The result is the DRS in (16). As the first disjunct in this DRS is negated, the shared conditions do not create an entailment relation between the clauses. Consequently, the disjunction is felicitous.

(15) Either there's no bathroom in this house, or it's in a strange place.

(16) I II $\neg[x][$ bathroom $(x)$, in-the-house $(x)] \vee[y][$ bathroom(y), in-thehouse(y), in-a-strange-place(y) ] ]

I do not wish to suggest that this anaphora strategy is governed by a strict rule. Essentially, it involves the selection by the hearer of some salient information for the interpretation of an otherwise uninterpretable definite. The default strategy is to look at the immediate linguistic context--the preceding clause--and to select as much relevant information as is available. Consequently, 
in the usual case, all of the descriptive content of the antecedent clause is used to construct an interpretation for the pronoun. This seems reasonable, given that the point of condition copying is to ensure that the new discourse referent will be mapped to the same individuals in the world as the old one; if they share all the same conditions, this will be guaranteed.

This, then, is all that is needed for the account of internal anaphora: the availability of an E-type strategy of pronoun interpretation, plus the recognition that disjunction itself is subject to certain constraints. We should observe, though, that the constraint on disjunction is more complex than I have suggested so far. (17) and (18) show a different pattern to the examples considered above:

\#Either Henry owns a car and Cleo owns a truck, or Henry owns a Japanese car (and Cleo doesn't own a truck).

(18) \#Either Henry lives in England and he owns a house, or he lives in London (and he doesn't own a house).

These examples show that in a disjunction whose disjuncts themselves consist of a conjunction of clauses, if any clause in one disjunct entails a clause in another disjunct, the disjunction is ruled out. Entailment between the disjuncts as a whole is not required in order for the disjunction to be ruled out; entailment between sub-parts of the disjuncts suffices.

There is surely some underlying principle at work here which produces these effects. In the scope of this paper, I cannot pursue the question of what exactly this principle might be. However, for our purposes here, the relevant observation is that the anaphora case behaves in just the same way:

\#Either Jane owns a bicycle and she's happy, or it's broken and she's unhappy.

This is predicted by my account, according to which the two cases are essentially identical. These more complicated examples thus provide further evidence that what has been thought of as an anaphora problem should be seen as a question about disjunction itself.

\section{External Anaphora}

Disjunctions of different syntactic types show slight differences with respect to external anaphora, but the same principles govern the anaphora in each case. I shall begin with the case of NP disjunction, and illustrate these principles. I will then go on to discuss clausal and VP disjunction. 


\subsection{NP disjunction}

As (20) and (21) illustrate, anaphora is possible between a disjunction of indefinite NPs in one sentence and a pronoun in the following sentence. The disjunctive NP may be in either subject or object position.

(20) Either a soprano or an alto will sing. She will stand on that platform. (21) George will sing either an aria or a ballad. It will have German lyrics.

The pronouns in these examples, though, are not anaphoric on either subordinate NP, but on the disjunctive NP as a whole. Consider (20). She cannot be interpreted as anaphoric on either a soprano or on an alto. It can only mean something like "whoever sings." I dub this the "whoever/whatever" interpretation.

It might be thought that anaphora to a subordinate NP is ruled out in (20) because of an unresolvable ambiguity: there is no way to determine whether the pronoun is intended as anaphoric on a soprano or on an alto, and so neither is possible. However, this reading is ruled out even when there is no potential ambiguity, as in (22).

(22) Either a soprano or a bass will sing. \#He will stand on that platform.

Any ambiguity would be resolved here by the gender clash between he and $a$ soprano, but nonetheless, the pronoun cannot be understood as anaphoric on $a$ bass. Moreover, as whoever sings may be either male or female, he cannot be given the "whoever/whatever interpretation." So the pronoun in this case is not felicitous under any interpretation. I have found, however, that speakers who generally accept they as a gender-neutral singular anaphor also accept (23) under the "whoever/whatever" interpretation.

(23) Either a soprano or a bass will sing. They'll stand on that platform.

Examples (20)-(22) contrast interestingly with cases in which one or both indefinites are replaced by proper names, as in (24)-(26). In these cases, the pronoun cannot be given a "whoever/whatever" interpretation, but it can now be interpreted as anaphoric on a particular subordinate NP, as long as there is no ambiguity.

(24) Either Jane or Maud will sing. \#She will stand on that platform.

(25) Either Jane or a soprano will sing. \#She has / will have a beautiful voice.

(26) a. Either Jane or George will sing. HE will also play the piano.

b. Jane and George will sing. HE will play the piano too.

To make this anaphoric reading natural, the pronoun needs to be slightly stressed. (Strong contrastive stress is not necessary; the pronoun simply must not be deaccented.) Whatever the reason for this, the same holds in the case of anaphora to a subordinate NP in a conjunction such as (26b). 
This pattern is not peculiar to proper names. Specific indefinites behave in just the same way:

(27) The concert will be opened by a soprano or an alto. She will stand on that platform.

(28) The concert will be opened by a famous mezzo who started her career as a violist, or a young soprano who recently sang at the Met. \#She will stand on that platform.

(29) The concert will be opened by a famous mezzo who started her career as a violist, or a young Welsh baritone who recently sang at the Met. HE is a very interesting performer.

How, then, are we to account for this range of possibilities?

The literature on anaphora already contains proposals which provide the formal machinery needed to account for the patterns observed. Rooth and Partee (1982) suggest that in certain cases, disjunctions introduce into a representation a single discourse referent, along with a disjunctive condition on that referent. Applying this proposal to the first sentence of (20) gives us the DRS in (30):

$$
[\mathrm{x}][[\operatorname{soprano}(\mathrm{x})] \vee[\operatorname{alto}(\mathrm{x})], \text { will-sing }(\mathrm{x})]
$$

The disjunction induces the introduction of a single discourse referent, $x$. This same referent occurs in the sub-DRS representing each disjunct. As $x$ is entered into the universe of the main DRS, it is accessible to pronouns in following sentences. This referent, though, represents neither a soprano nor an alto; it represents whoever sings. Any pronoun interpreted using this referent will therefore have this interpretation. Anaphora to one of the subordinate NPs is impossible because neither introduces its own discourse referent. ${ }^{6}$

To explain the proper name case, we can adopt the assumption of Kamp and Reyle (1993) that proper names and specific indefinites always induce the introduction of a discourse referent into the main DRS, even when they occur in a subordinate position. Assuming that the same applies to proper names inside a disjunction, the DRS for the first sentence of (26a) will be as in (31):

$$
[\mathrm{x}, \mathrm{y}][\mathrm{x}=\text { Jane, } \mathrm{y}=\text { George, [will-sing }(\mathrm{x})] \vee[\text { will-sing }(\mathrm{y})]]
$$

Here, the NPs behave just as they would in the absence of disjunction. Each introduces its own discourse referent into the universe of the main DRS, and both of these referents are accessible to following pronouns. If a following pronoun is equated with $x$, it will be interpreted as anaphoric on Jane; if equated with $y$, it will be anaphoric on George. In this representation, there is no referent which represents the disjunction as a whole, and consequently no "whoever/whatever" reading is available for a following pronoun.

By assuming that the representation of the examples with non-specific indefinites differs from that of examples with proper names and specific indefinites, we can provide a formal account of the various readings available. 
But on what basis can we motivate this assumption? To see this, we need to consider what these representations really express.

The introduction of a discourse referent into the universe of the main DRS represents the fact that the speaker--and by extension, the hearer--is committed to the existence of an individual satisfying certain properties. The properties are given by the conditions associated with that referent. Discourse referents introduced into the universe of a sub-DRS represent entities that play a role in the discourse, but to whose existence the speaker is not committed. ${ }^{7}$

In asserting a disjunction which contains indefinites, there are a number of different commitments which a speaker might make with respect to the existence of individuals. Suppose that, on the basis of a particular experience, I say:

A soprano or an alto was singing. She had a lovely voice.

This assertion is based on a belief about a particular individual--the individual who was singing. The disjunction indicates my uncertainty about the appropriate characterization of this individual. In making the assertion, I commit myself to the existence of only one individual. This is represented by the introduction into the main DRS of a single discourse referent.

Similarly, suppose that I am telling you about a concert which is to take place. The concert is a regular event, at which either a soprano or an alto always sings. I have no knowledge about who the performer might be, but I do know that there will be a performer satisfying one of these descriptions. In these circumstances, my assertion:

A soprano or an alto will sing. She will have a lovely voice.

again commits me to the existence of only one individual, while offering alternative possible characterizations of that individual. So again, the disjunction is appropriately represented by a single discourse referent in the universe of the main DRS, along with a disjunctive condition.

Suppose, though, that I say:

Either Cleo or Maud will sing.

By saying this, I commit myself to the existence of two separate individuals--Cleo and Maud--and indicate that one of them will satisfy the predicate given. There is no other way to understand the disjunction. To represent this commitment, we must introduce two distinct referents into the main DRS, one for Maud and one for Cleo.

The use of a disjunction of specific indefinites carries just the same commitment. A specific indefinite is an indefinite whose descriptive content is such that an assertion containing this indefinite must be based on a belief about a particular individual, just like an assertion containing a proper name. An assertion of (28), repeated here, commits the speaker to the existence of 
individuals satisfying each of the descriptions. Its representation must therefore include a discourse referent for each of these individuals.

(28) The concert will be opened by a famous mezzo who started her career as a violist, or a young soprano who recently sang at the Met.

The possibilities of external anaphora to NP disjunctions are therefore determined by the role these expressions play in introducing individuals into the discourse context. A disjunction of non-specific indefinites will, in general, serve to introduce a single individual under alternative characterizations. This is represented by introducing a single discourse referent into the main DRS. This referent is accessible to following pronouns, and gives rise to the "whoever/whatever" interpretation. On the other hand, disjunctions of proper names or specific indefinites introduce (at least) two individuals, and assert that (at least) one of them will satisfy the predicate given. This is represented by introducing a distinct referent for each NP into the main DRS. Each of these referents can be used to interpret a following pronoun, and will give rise to an anaphoric link to a particular subordinate NP.

\subsection{Clausal Disjunction}

The pattern of anaphora to clausal disjunctions is very similar to what we have already seen. Clausal disjunctions which contain indefinites support external anaphora, but the pronoun is given a "whoever/whatever" interpretation (examples (35)-(37)). If the indefinites are replaced with proper names, or with specific indefinites, the "whoever/whatever" reading disappears, but, as with the NP disjunctions, the pronoun may be understood as anaphoric on a particular NP in one of the disjuncts (examples (38)-(40)).

(35) For the final act, either a soprano will sing or an actress will perform a monologue. She will stand on that platform.

(36) Either a squirrel has got into the attic, or a bird is building a nest up there. We'll have to get it out.

(37) Either Jane talked to a bass about this, or Cleo talked to a tenor. He was very upset.

(38) Either Jane will sing or Maud will play the piano. \#Then she'll lead the audience in the national anthem.

(39) Either a famous mezzo who started her career as a violist will sing, or a young pianist who won the Rubinstein competition a couple of years ago will play. \#She'll probably do several encores.

(40) Either Jane will sing or George will play the piano. HE is a very interesting performer.

These disjunctive sentences serve to introduce individuals into the context in much the same way as the NP disjunctions do. The first sentence of (35) 
presents two alternative situations, and asserts that (at least) one of them will come about. However, the sentence commits the speaker to the existence of only one individual, the performer. (If the disjunction is verified by the truth of the first disjunct, the speaker has no commitment to the existence of an actress.) The natural way to represent this sentence, then, is with the DRS in (41):

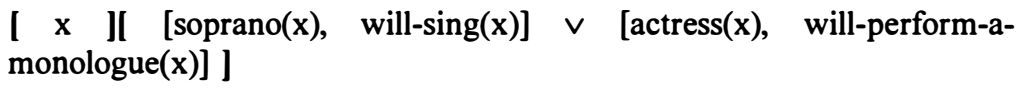
monologue(x)] ]

As in the case of NP disjunction, this representation provides a single variable for the interpretation of following pronouns. This variable represents neither a soprano nor an actress, but the individual whose existence will satisfy the disjunctive condition. A pronoun interpreted using this variable will therefore be understood as "whoever performs." Note, though, that to derive this representation, we must suspend, or at least loosen, the novelty requirement on referents introduced by indefinites. I return to this point below.

(40) also presents two alternative situations, but here, each alternative involves a distinct individual. The speaker of this sentence is committed to the existence of both Jane and George, and so its representation must include discourse referents for both. The DRS for this sentence is shown in (42).

$$
[x, y][x=\text { Jane, } y=\text { George, }[\text { will-sing }(x)] \vee[\text { will-play }(y)]]
$$

Not all clausal disjunctions containing indefinites support external anaphora, even with a "whoever/whatever" reading. When the indefinites are in the sentence comment, the felicity of the anaphora is often considerably reduced. Consider, for example, (43)-(45):

(43) Either Cleo has baked a cake, or Henry has made a trifle. \#They're both excellent cooks, so I'm sure it's delicious.

(44) Either a soprano will sing an aria, or an actress will recite a monologue.

a. Then she will lead the audience in the national anthem.

b. ?It'll be in German.

(45) Either an aria will be sung by a soprano, or a monologue will be recited by an actress.

a. \#Then she will lead the audience in the national anthem.

b. It'll be in German.

This is not, however, a straightforward subject/object asymmetry, as illustrated by the felicity of (46) and (47):

(46) Either George will send an e-mail, or Maud will write a letter. It'll contain all of the relevant information.

(47) Jane had a terrible time getting to Boston. Either she had rented a car, or Henry had lent her a truck, but it broke down on the way. 
The reason for this asymmetry is, I think, that a disjunctive sentence is often not interpreted as presenting alternative characterizations of indefinites in the comment. (43), intuitively, is not about a single dessert; its function is not to introduce some entity (a dessert), but to talk about Cleo and Henry. It is not, though, impossible for indefinites in the comment to function in this way. (46) is naturally understood as giving information about a single communication, which will be either a letter or an e-mail. Consequently (46), but not (43), allows the anaphora.

As in the case of proper names and specific indefinites, the absence of a "whoever/whatever" reading in examples like (43) is expressed formally by introducing separate discourse referents for each indefinite. The impossibility of anaphora to the subordinate NPs indicates, though, that these referents cannot be introduced into the main DRS. This accords with the observation that the referents do not represent entities to whose existence the speaker is committed. In this case, each referent is introduced into the universe of the sub-DRS representing the disjunct in which it occurs. The complete DRS for (43) is given in (48):

$$
\begin{aligned}
& {[x, y][x=\text { Cleo, } y=\operatorname{Henry,}[[u][\operatorname{cake}(u), x \text { baked } u] \vee[v][\operatorname{trifle}(v), y} \\
& \text { made } v]]]
\end{aligned}
$$

\subsubsection{Modal Subordination Readings}

There is one other case of external anaphora to a clausal disjunction containing indefinites which is not available with NP disjunction. This is the case of modal subordination, as discussed by Roberts (1989). An example is given in (49):

(49) Either a soprano will sing or an actress will perform a monologue. But SHE would have to give a really impressive performance.

The pronoun she (which, I think, must be stressed), is understood as anaphoric on the indefinite an actress, although this indefinite is not specific. But the sentence containing the pronoun is, as a whole, subordinate to the second disjunct, meaning something like: "If it's the actress who performs, then she will have to give a really impressive performance." ${ }^{8}$ This implicit conditional is understood as being conjoined to the second disjunct.

In (48), the indefinites in each disjunct introduce their referents into the universe of the sub-DRS representing that disjunct. Modal subordination can be accounted for formally by allowing any indefinite in a clausal disjunction to introduce a referent in this way. This would produce the DRS in (50) as the representation of the first sentence of (49):

(50) [ ][ [[x][soprano(x), will-sing(x)]] $\vee[[y][\operatorname{actress}(y)$, will-perform-amonologue(y)]] ] 
Let us assume (more or less following Roberts) that the subordination of the second sentence in the sequence to the second disjunct of (50) is expressed by conjoining the two. The second sentence thus becomes part of this disjunct, and not part of the main DRS. The definites in this sentence will thus be subordinate to the referent $y$ in the universe of the second disjunct, and so can be interpreted via this referent. Consequently, they are interpreted as anaphoric on an actress.

Recall that the first sentence of (49) also supports external anaphora with a "whoever/whatever" reading. To account for this, I argued above that the appropriate DRS for this sentence is that shown in (51), repeated from above:

$$
\begin{aligned}
& {[\quad x \quad \text { II }[\operatorname{soprano}(x) \text {, will-sing }(x)] \vee \text { [actress(x), will-perform-a- }} \\
& \text { monologue(x)] ] }
\end{aligned}
$$

(50) and (51) are truth-conditionally equivalent; both will be verified in the same models. They are not, of course, dynamically equivalent. (51) allows external anaphora, but (50) does not. (50), on the other hand, can quite straightforwardly be derived compositionally from the syntactic representation of the disjunction, whereas (51) cannot. To derive (50), we map each syntactic disjunct into a subDRS of the disjunctive condition, and then reduce each clause following the standard DRT construction rules. To derive (51), we would need to modify these rules, including, as I mentioned earlier, the novelty requirement for indefinites.

Consequently, I take (50) to be the default, and basic, representation for the sentence, from which (51) can be derived. Derivation of the reduced representation will follow from recognition that the sentence is being used to introduce a single individual under alternative characterizations. There is thus a significant pragmatic input at this stage. This pragmatic factor explains the variability in speaker judgements about external anaphora to clausal disjunctions. Some speakers are much more willing to accept this kind of anaphora than others. Similarly, some examples are much more acceptable than others with identical structure but different content. In general, the more obviously the NP predicates are related, the more acceptable the anaphora is. Here, for instance, is an example structurally identical to the first sentence of (49) which does not seem to support external anaphora at all:

(52) Either a car just backfired, or a gun went off.

a. ...\#It must have been a small one.

b. ...\#I think it belongs to George.

The problem seems to be that it is quite unnatural to generalize over cars and guns, and so to understand the sentence as of fering alternative characterizations of a single object.

\subsection{VP Disjunction}

Anaphora to VP disjunction, as might be expected, follows the same pattern as anaphora to clausal disjunctions where the relevant NPs form part of the sentence 
comment. It is easier to find unacceptable examples than acceptable ones; the acceptable cases are those where the VPs provide alternative characterizations of a single entity.

(53) Jane will either buy a bicycle or borrow a motorbike. \#It'll be really useful for getting around.

(54) Maud will either make a trifle or buy a cake. \#It'll be great for dessert.

(55) Jane either bought a bicycle or borrowed a bicycle. It's in the garage.

All of the examples are improved by deaccenting the NP, and concomitantly stressing the verb. In general, deaccenting indicates that an NP is "old information". In (53), for instance, the effect of deaccenting is to indicate that it is taken for granted that Jane will acquire some vehicle or other, and what is of interest is how this will happen. With deaccenting, it is easier to understand the sentence as giving alternative characterizations of the vehicle.

I assume that, as with clausal disjunction, VP disjunctions trigger the introduction of a disjunctive condition, with each disjunct being mapped to one sub-DRS, and the indefinites introducing their discourse referents into the universe of the relevant sub-DRS. The DRS for the first sentence of (53) is given in (56):

$[\mathrm{x}$ II $\mathrm{x}=$ Jane, $[\mathrm{y}][\operatorname{bicycle}(\mathrm{y}), \mathrm{x}$ will buy $\mathrm{y}] \vee[\mathrm{z}][\operatorname{motorbike}(\mathrm{z}), \mathrm{x}$ will borrow z] ]

Again like the clausal disjunctions, if a sentence containing a VP disjunction is understood as giving alternative characterizations of a single entity, the representation may be modified to reflect this, producing, for (53), the DRS in (57):

[ $\mathrm{x}, \mathrm{y}][\mathrm{x}=$ Jane, [bicycle $(\mathrm{y}), \mathrm{x}$ will buy $\mathrm{y}] \vee[$ motorbike(y), $\mathrm{x}$ will borrow $y$ ] ]

\subsection{External Anaphora: Summary}

(i) External anaphora with "whoever/whatever" reading

Produced by anaphora to a discourse referent introduced into the universe of the main DRS, and occurring on both sides of the disjunctive condition. A disjunction gives rise to such a referent when it is understood to introduce a single individual under alternative characterizations.

(ii) External anaphora to a subordinate $N P$

Possible only with proper names and specific indefinites, which always introduce a discourse referent into the universe of the main DRS. Disjunctions containing NPs of this type are always understood as introducing multiple individuals, and asserting that (at least) one of them will satisfy the given predicate. 


\section{(iii) No external anaphora}

Results when the referent introduced by each indefinite NP is introduced into the universe of the sub-DRS representing the disjunct in which it occurs. This is the default representation for indefinites in clausal and VP disjunctions, from which reduced representations as specified in (i) can be derived. ${ }^{9}$ These default representations do support external anaphora in the case of modal subordination, where the sentence containing the pronoun is conjoined to one of the disjuncts, making the referents in its universe accessible to the pronoun.

\section{Conclusion}

As I said at the outset, distinct accounts are needed for internal and external anaphora. Both accounts, however, make reference to the function of disjunction itself. Recognition of this function is particularly important in the analysis of internal anaphora, where it is not the anaphora itself which causes infelicity, but the failure of the disjunction to present "true alternatives" when an anaphoric link is formed across disjuncts. Previous analyses have attempted to account for these data by explaining what is wrong with the anaphora in the bad examples. The explanation has been very hard to find. The reason is that we have been asking the wrong question. When we ask the right one--what is wrong with the disjunction?--a quite natural answer is forthcoming.

\section{Endnotes}

- For their help and encouragement in writing this paper, I would like to thank Molly Diesing, Sally McConnell-Ginet, Zoltán Gendler Szabó and Sandro Zucchi. Thanks also to the participants of SALT VI for helpful and interesting discussion. 1. In this paper, I discuss only internal anaphora between clausal disjuncts. However, similar phenomena arise with disjunctions of other categories.

2 . \# indicates infelicity of a sentence, or of a sentence in a particular sentence sequence.

3. Jeff Pelletier has brought to my attention a similar claim made in Hurford (1974). Hurford claims that "the joining of two sentences by or is unacceptable if one sentence entails the other; otherwise, the use of or is acceptable." Hurford's formulation is problematic in two respects. First, there are sentences in which or is not used to express disjunction; these sentences are not subject to the disjunction constraint. Second, as I will illustrate below, Hurford's biconditional formulation is too strong.

4. The idea of incorporating an E-type strategy into a dynamic framework does not originate with me. Chierchia (1995) assumes both a pragmatic E-type strategy as well as dynamic binding. Kamp and Reyle (1993) use a very similar strategy to that which I propose as part of their account of plural anaphora to quantificational NPs. DRT accounts of anaphora which make use of 
accommodation (see, for instance, Kadmon (1987) and Roberts (1989)) likewise involve very similar mechanisms.

5. I hope at some later point to show that these accessibility relations follow from general principles governing information representation and update. For now, I follow Kamp and Reyle in stipulating them.

6. Recall that I characterized the E-type strategy proposed above as a rescue strategy, which comes into play when there is no accessible referent. Here there is an accessible referent, so there is no "license" to use the E-type strategy to construct an alternative interpretation.

7. Cf. the distinction made in Roberts (1989) between factual and non-factual modalities.

8. As Roberts points out, the subordination is indicated by the past tense modal.

9. We could make the same assumption about NP disjunctions. At this stage, I remain agnostic on this point.

\section{References}

Chierchia, Gennaro (1992). "Anaphora and Dynamic Binding." Linguistics and Philosophy 15: 111-183.

Chierchia, Gennaro (1995). Dynamics of Meaning: Anaphora, Presuppositions and the Theory of Grammar. Chicago: University of Chicago Press.

Evans, Gareth (1977). "Pronouns, Quantifiers and Relative Clauses". Canadian Journal of Philosophy Vol. VII(3) 467-536.

Evans, Gareth (1980). "Pronouns". Linguistic Inquiry Vol.II(2) 337-362.

Groenendijk, Jeroen and Martin Stokhof (1990). "Dynamic Montague Grammar." In Laszlo Kalman and Laszlo Polos (eds.), Papers from the Second Symposium on Logic and Language. Budapest: Akademiai Kiado.

Groenendijk, Jeroen and Martin Stokhof (1991). "Dynamic Predicate Logic." Linguistics and Philosophy 14: 39-100.

Heim, Irene (1982). The Semantics of Definite and Indefinite Noun Phrases, PhD Thesis, University of Massachusetts, Amherst. Distributed as Arbeitspapier 73, SFB 99, Konstanz.

Heim, Irene (1990). "E-type Pronouns and Donkey Anaphora." Linguistics and Philosophy 13: 137-177.

Hurford, James R. (1974). "Exclusive and Inclusive Disjunction." Foundations of Language 11: 409-411.

Kadmon, Nirit (1987). On Unique and Non-Unique Reference and Asymmetric Quantification. PhD Thesis, UMass.

Kamp, Hans (1981). "A Theory of Truth and Semantic Representation". In: Jeroen A.G. Groenendijk, T.M.V. Janssen \& Martin B.J. Stockhoff (eds.), Truth, Representation and Information (=GRASS Series No.2), Dordrecht, pp.277-322.

Kamp, Hans and Uwe Reyle (1993). From Discourse to Logic. Boston: Kluwer Academic Press. 
Neale, Stephen (1990). Descriptions. Cambridge, Mass.:MIT Press.

Roberts, Craige (1989). "Modal Subordination and Pronominal Anaphora in Discourse." Linguistics and Philosophy 12(6): 683-721.

Rooth, Mats and Barbara Partee (1982). "Conjunction, Type Ambiguity and Wide Scope 'Or'," in D. Flickenger, M. Macken and N. Wiegand (eds.), Proceedings of the First West Coast Conference on Formal Linguistics. Linguistics Department, Stanford University.

Stalnaker, Robert (1978). "Assertion." In P. Cole (ed.), Syntax and Semantics Vol. 9. New York: Academic Press.

Stone, Mathew (1992). "Or and Anaphora." In Chris Barker and David Dowty (eds.), SALT II. Linguistics Department, Ohio State University. 